\title{
Special Issue "Information Technology for Geotechnical Engineering”
}

\author{
Kok-Kwang Phoon $\cdot$ Siang Huat Goh
}

Published online: 15 March 2013

(C) Springer Science+Business Media Dordrecht 2013

The application of information technology to geotechnical engineering is not new. The storage of borehole information in a simple electronic form has evolved rapidly over the years to take advantage of numerous emerging technologies such as the World Wide Web and the geographical information system. There are also increasing attempts to integrate geoinformation from all available sources such as geophysical, geological, and remote sensing databases for surface and subsurface modeling as well as with data from downstream applications such as monitoring data from construction management of large projects or monitoring data for hazard/disaster management. The usefulness of electronic databases is also augmented by advancement in sensor technology (related to upstream quality and quantity of data) and decision support systems (related to downstream interpretation of data).

The special issue contains nine invited papers. The papers are arranged according to the last name of the first author. Eight of them were presented at the International Symposium on Advances in Ground Technology and Geo-Information (IS-AGTG), which was held in Singapore between 1 and 2 December 2011. The guest editors are grateful to Professor Vassilis Marinos and his co-workers for contributing an additional paper to this special issue.

K.-K. Phoon $(\varangle) \cdot$ S. H. Goh

National University of Singapore, Singapore, Singapore

e-mail: kkphoon@nus.edu.sg
Among the nine invited papers, two papers discussed the exploitation of information systems for tunneling projects (Chmelina et al. and Marinos et al.). Chmelina et al. described the application of an information system that integrates site data for monitoring and early warning at four tunneling projects, namely Metro Thessaloniki, Metro Budapest, Cityringen Copenhagen and Crossrail London. Marinos et al. described the development of a database named Tunnel Information and Analysis System (TIAS) based on geological, geotechnical, and structural information associated with 62 road tunnels in northern Greece.

Two papers highlighted the potential to improve project management in a data rich environment for groundwater remediation (Miyata and Hata) and deep excavation (Rackwitz et al.). Miyata and Hata discussed the usefulness of information technology for monitoring contaminants and groundwater level, transferring data between the remediation well and the remote remediation center, and hosting a fuzzy decision support system for controlling the remediation well. Rackwitz et al. discussed the development of a web-based software platform DoMaMoS to support routine construction operations and to handle exceptional situations that can lead to disasters. The platform is applied to the "Spreedreieck" excavation project in Berlin to showcase its project/quality management and risk management capabilities.

Two papers (Inoue et al. and Todo et al.) discussed the application of databases for subsurface mapping. 
Inoue et al. described how a three-dimensional geological map for the Kansai International Airport, Japan, could be updated by incorporating data from a $1,300 \mathrm{~m}$ deep borehole and seismic profiles. Todo et al. presented an effort by the Japanese Geotechnical Society (JGS) to construct a "Nation-wide Electronic Geotechnical Database Systems" (NEGDS), which can electronically construct ground models of $250 \mathrm{~m}$ by $250 \mathrm{~m}$ in plan and up to $100 \mathrm{~m}$ in depth.

Two papers discussed the application of artificial intelligence techniques to produce data-driven models (Gomes Correia et al. and Yoo). Gomes Correia et al. demonstrated the applications of artificial intelligence techniques, such as data mining (artificial neural networks and support vector machines) and evolutionary computation, for earthwork management, compaction, pavement evaluation, design and management, and jet grouting. Yoo proposed a novel approach to calibrate artificial neural networks
(ANNs) using finite element analyses to serve as an inexpensive prediction tool. The calibrated ANNs are implemented on a GIS platform for visualizing and analyzing the spatial distribution of predicted results. The proposed approach was applied to a soft ground improvement project in the Gwang Yang area, located in the southern part of Korea, and design of a support system for a hypothetical underground cavern.

Last but not least, Abdoun et al. presented a paper on sensor development. The Shape Acceleration Array (SAA) system is found to be capable of measuring in situ deformations and accelerations up to a depth of $100 \mathrm{~m}$ for health monitoring of aging civil infrastructure in the United States.

We are grateful to Professor Paul Marinos for supporting this Special Issue. We would also like to thank the authors for their efforts to prepare the papers and the reviewers for their time to provide constructive comments. 